\title{
Homeostatic Plasticity and External Input Shape Neural Network Dynamics
}

\author{
Johannes Zierenberg, ${ }^{1,2, *}$ Jens Wilting, ${ }^{1, *}$ and Viola Priesemann ${ }^{1,2}$ \\ ${ }^{1}$ Max Planck Institute for Dynamics and Self-Organization, Am Fassberg 17, 37077 Göttingen, Germany, \\ ${ }^{2}$ Bernstein Center for Computational Neuroscience, Am Fassberg 17, 37077 Göttingen, Germany,
}

(Received 3 August 2017; revised manuscript received 8 June 2018; published 20 July 2018)

\begin{abstract}
In vitro and in vivo spiking activity clearly differ. Whereas networks in vitro develop strong bursts separated by periods of very little spiking activity, in vivo cortical networks show continuous activity. This is puzzling considering that both networks presumably share similar single-neuron dynamics and plasticity rules. We propose that the defining difference between in vitro and in vivo dynamics is the strength of external input. In vitro, networks are virtually isolated, whereas in vivo every brain area receives continuous input. We analyze a model of spiking neurons in which the input strength, mediated by spike rate homeostasis, determines the characteristics of the dynamical state. In more detail, our analytical and numerical results on various network topologies show consistently that under increasing input, homeostatic plasticity generates distinct dynamic states, from bursting, to close-to-critical, reverberating, and irregular states. This implies that the dynamic state of a neural network is not fixed but can readily adapt to the input strengths. Indeed, our results match experimental spike recordings in vitro and in vivo: The in vitro bursting behavior is consistent with a state generated by very low network input $(<0.1 \%)$, whereas in vivo activity suggests that on the order of $1 \%$ recorded spikes are input driven, resulting in reverberating dynamics. Importantly, this predicts that one can abolish the ubiquitous bursts of in vitro preparations, and instead impose dynamics comparable to in vivo activity by exposing the system to weak long-term stimulation, thereby opening new paths to establish an in vivo-like assay in vitro for basic as well as neurological studies.
\end{abstract}

DOI: 10.1103/PhysRevX.8.031018

\section{INTRODUCTION}

Collective spiking activity clearly differs between in vitro cultures and in vivo cortical networks (see examples in Fig. 1). Cultures in vitro typically exhibit stretches of very little spiking activity, interrupted by strong bursts of highly synchronized or coherent activity [1-7]. In contrast, spiking activity recorded from the cortex in awake animals in vivo lacks such pauses, and instead shows continuous, fluctuating activity. These fluctuations show a dominant autocorrelation time that was proposed to increase hierarchically across the cerebral cortex, from sensory to frontal areas [8]. Moreover, depending on experimental details such as the brain area, species, and vigilance state, one also observes evidence for asynchronous-irregular (AI) dynamics [9,10]; oscillations [11-13]; or strong fluctuations associated with criticality, bistability, or up-and-down states [14-20]. These states differ not only in strength and structure of

*J. Z., and J. W. contributed equally to this work.

Published by the American Physical Society under the terms of the Creative Commons Attribution 4.0 International license. Further distribution of this work must maintain attribution to the author(s) and the published article's title, journal citation, and DOI.
Subject Areas: Biological Physics, Complex Systems, Interdisciplinary Physics fluctuations, but also in synchrony among neurons, from uncorrelated to fully synchronized spiking. The observation of such a vast range of dynamic states is puzzling, considering that the dynamics of all networks presumably originate from similar single-neuron physiology and plasticity mechanisms.

One particular plasticity mechanism that regulates neural activity on a long timescale is homeostatic plasticity [23-28]. Homeostatic plasticity can be implemented by a number of physiological candidate mechanisms, such as redistribution of synaptic efficacy [29,30], synaptic scaling [23-25,31], adaptation of membrane excitability [27,32], or through interactions with glial cells [33,34]. All these mechanisms have in common that they implement a slow negative feedback loop in order to maintain a certain target spike rate and stabilize network dynamics. In general, they reduce (increase) excitatory synaptic strength or neural excitability if the spike rate is above (below) a target rate, allowing compensation against a potentially unconstrained positive feedback loop through Hebbian-type plasticity [35-41]. Recent results highlight the involvement of homeostatic plasticity in generating robust yet complex activity dynamics in recurrent networks [42-44].

To understand the physiological mechanisms behind this large set of dynamic states, different model networks have been proposed that reproduce one or a set of states. 
To name a few examples, deafferentiation in combination with homeostatic scaling can generate bursts [45]; the interplay between excitation and inhibition may lead to oscillations, synchronous-regular activity, or asynchronousirregular activity [46-49], where switching between dynamic states can be induced by varying the input [49-51]; synaptic facilitation and depression promote regular and irregular network dynamics [52-54]; plasticity at inhibitory synapses can stabilize irregular dynamics $[55,56]$, whereas specific types of structural [57-59] or synaptic [53,54,60-68] plasticity foster strong temporal fluctuations characteristic for a critical state; and last but not least, homeostasis is necessary to achieve stable dynamics in recurrent networks with spiketiming dependent plasticity (STDP) or Hebbian-type synaptic plasticity (e.g., Refs. [40,69-73]). Overall, the dynamic state depends on all aspects: single-neuron properties, synaptic mechanisms, network topology, plasticity rules, and input characteristics. Recalling that the single-neuron properties, synaptic mechanisms, and plasticity rules are presumably very similar in vitro and in vivo, these factors are unlikely to explain the observed differences.

In this study, we propose that the input strength is the defining difference between in vitro and in vivo dynamics. In vitro systems are completely isolated, whereas in vivo networks receive continuous input from sensory modalities and other brain areas. Under these different conditions, we propose that homeostatic plasticity is a sufficient mechanism to promote self-organization to a diverse set of dynamic states by mediating the interplay between external input rate and neural target spike rate. Treating the external input as a control parameter in our theoretical framework allows us to alter the network dynamics from

\section{in vitro}
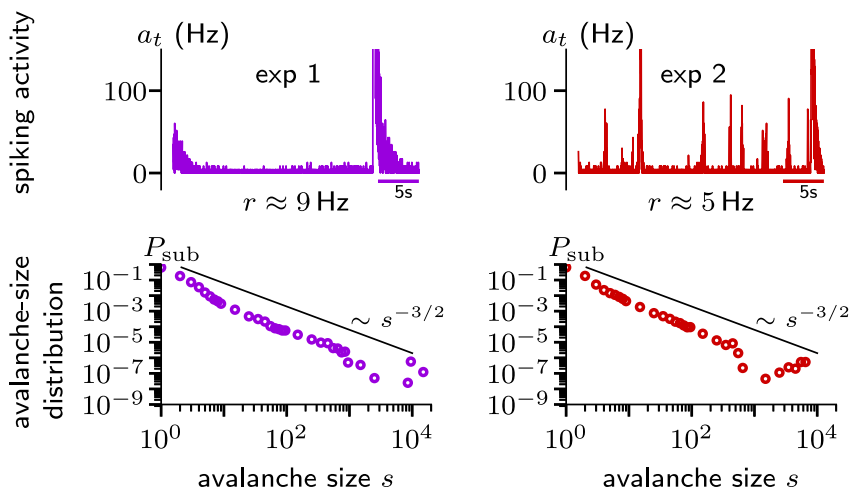

in vivo
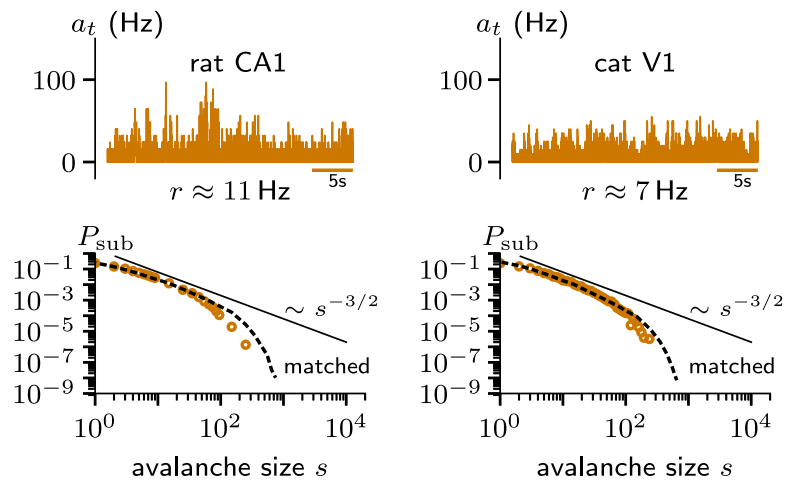

FIG. 1. Examples of dynamic states observed in experiments. In vitro spike recordings are from cultures of dissociated rat cortical neurons [4]. In vivo recordings are from the right dorsal hippocampus in an awake rat during an open field task [21] and from the primary visual cortex in a mildly anesthetized cat [22]. The top row shows population spiking activity (Appendix B-1) from 30-60 single or multiunits ( $\Delta t=4 \mathrm{~ms}$ ), with average neural firing rate $r$; the bottom row shows subsampled avalanche-size distributions (Appendix B-2). Solid lines indicate the power-law behavior $s^{-3 / 2}$ expected for a critical branching process. Dashed lines correspond to distributions obtained from branching networks matched to the experiments of rat CA1 ( $\tau=2 \mathrm{~s}, r^{*}=11 \mathrm{~Hz}, h=5.5 \times 10^{-1} \mathrm{~Hz}, n=31, N=10^{4}$, $\left.\Delta t=1 \mathrm{~ms}, \tau_{\mathrm{hp}}=10^{5} \mathrm{~s}\right)$ and cat V1 $\left(\tau=0.2 \mathrm{~s}, r^{*}=7 \mathrm{~Hz}, h=3.5 \times 10^{-2} \mathrm{~Hz}, n=50, N=10^{4}, \Delta t=1 \mathrm{~ms}, \tau_{\mathrm{hp}}=10^{5} \mathrm{~s}\right)$. For details and a definition of parameters, see Appendix B-4. 
by periods of synchronized activity. The subsampled avalanche-size distributions $P_{\text {sub }}(s)$ exhibit partial powerlaw behavior resembling $P(s) \sim s^{-3 / 2}$, as expected from a critical branching process [77] and conjectured for the synchronous-irregular regime [78]. However, in addition, $P_{\text {sub }}(s)$ also shows a peak at large avalanche sizes, which may indicate either finite-size effects, supercriticality, or characteristic bursts [79].

In contrast, the spiking activity in vivo shows fluctuating dynamics (Fig. 1). These have been described as reverberating dynamics, a dynamic state between critical and irregular dynamics [80], characterized by a finite autocorrelation time of a few hundred milliseconds. The subsampled avalanchesize distributions $P_{\text {sub }}(s)$ can be approximated by a power law for small $s$ but show a clear exponential tail. The tails indicate slightly subcritical dynamics [81], especially because deviations in the tails are amplified under subsampling $[15,16,79]$.

In sum, the spiking activity and the corresponding avalanche-size distributions clearly differ between in vitro and in vivo recordings. Remarkably, however, the average neural firing rate $r$ is similar across the different experimental setups.

\section{MODEL}

To investigate the differences between in vitro and in vivo, we make use of a branching network, which approximates properties of neural activity propagation. We extend the branching network by a negative feedback, which approximates homeostatic plasticity.

\section{A. Branching network}

In the brain, neurons communicate by sending spikes. The receiving neuron integrates its input, and if the membrane potential crosses a certain threshold, this neuron fires a spike itself. As long as a neuron does not fire, its time-varying membrane potential can be considered to fluctuate around some resting potential. In the following, we approximate the complex time-resolved process of action potential generation and transmission in a stochastic neural model with probabilistic activation.

Consider a network of size $N$. Each node corresponds to an excitatory neuron, and spike propagation is approximated as a stochastic process at discrete time steps $\Delta t$. If a neuron, described by the state variable $s_{i, t} \in\{0,1\}$, is activated, it spikes $\left(s_{i, t}=1\right)$ and immediately returns to its resting state $\left(s_{i, t+1}=0\right)$ in the next time step, unless activated again. Furthermore, it may activate postsynaptic neurons $j$ with probability $p_{i j, t}=w_{i j} \alpha_{j, t}$, where $w_{i j} \in$ $\{0,1\}$ indicates whether two neurons are synaptically connected, and $\alpha_{j, t}$ is a homeostatic scaling factor. In addition, each neuron receives network-independent external input at rate $h_{i}$, representing external input from other brain areas, external stimuli, and importantly also spontaneous spiking of single neurons generated independently of presynaptic spikes (e.g., by spontaneous synaptic vesicle release $[82,83])$. The uncorrelated external input homogeneously affects the network at rate $h_{i}=h$, modeled as Poisson processes with an activation probability $1-e^{-h \Delta t} \simeq h \Delta t$.

This model can be treated in the framework of a branching process [77], a linear process with a characteristic autocorrelation time $\tau$ (see below). The population activity is characterized by the total number of spiking neurons, $A_{t}=\sum_{i=1}^{N} s_{i, t}$. Each spike at time $t$ generates, on average, $m$ postsynaptic spikes at time $t+1$ such that, on average, $\mathbb{E}\left(A_{t+1} \mid A_{t}\right)=m A_{t}$, where $m$ is called the "branching parameter." The branching parameter can be defined for each neuron individually: Neuron $i$ activates, on average,

$$
m_{i, t}=\sum_{j=1}^{N} w_{i j} \alpha_{j, t}
$$

of its postsynaptic neurons [84]. This local branching parameter $m_{i, t}$ thus quantifies the impact of a spike in neuron $i$ on the network. The network average (denoted in the following with a bar) of $m_{i, t}$ generates the (timedependent) network branching parameter [67]

$$
\bar{m}_{t}=\frac{1}{N} \sum_{i=1}^{N} m_{i, t} .
$$

The external input generates, on average, $N h \Delta t$ additional spikes per time step, resulting in a driven branching process $[85,86]$. The expected activity at time $t+1$ is then $\mathbb{E}\left(A_{t+1} \mid A_{t}\right)=m A_{t}+N h \Delta t$. For $m<1$, the process is called "subcritical," meaning that individual cascades of events will eventually die out over time. In this case, the temporal average (denoted in the following as $\langle\cdot\rangle$ ) of network activity $A_{t}$ converges to a stationary distribution with average activity

$$
\langle A\rangle=\frac{1}{T} \sum_{t=1}^{T} A_{t} \underset{T \rightarrow \infty}{\rightarrow} \frac{N h \Delta t}{1-m} .
$$

Considering a homogeneous neural spike rate $r_{i}=r=$ $\langle A\rangle / N \Delta t$, this implies

$$
r=\frac{h}{1-m} .
$$

A constant mean spike rate $r$, which can be considered a biological constraint, is thus realized by adjusting either $m \in[0,1)$ or $h \in[0, \infty)$.

The subcritical branching process $(m<1)$ is stationary with the autocorrelation function $C(l)=m^{l}$. The autocorrelation time can be identified by comparing with an exponential decay $C(l)=e^{-l \Delta t / \tau}$, yielding [80] 


$$
\tau=-\Delta t / \ln (m),
$$

which diverges as $m \rightarrow 1$. At this divergence $(m=1)$, the process is critical and the activity $A_{t}$ grows linearly in time with rate $h$. At criticality, assuming $h \rightarrow 0$, the number of events $s$ in an avalanche triggered by a single seed event is distributed according to a power law $P(s) \sim$ $s^{-3 / 2}$ [77]. For a nonvanishing $h$ in the subcritical regime $(m<1)$, the avalanche-size distributions show a rapid decay, if they can be measured at all under persistent activity (Appendix B-2). Finally, for $m>1$, the process is called "supercritical," and $A_{t}$ can, in principle, grow to infinity. For a finite network, this, of course, is not possible and will manifest in a peak of the avalanchesize distribution at large avalanche sizes.

For the computational model, we consider a network of $N=10^{4}$ neurons, which represents the size of in vitro cultures and in vivo cortical hypercolumns. The time step of the branching process has to reflect the causal signal propagation of the underlying physiological network. Since realistic propagation times of action potentials from one neuron to the next range from 1-4 $\mathrm{ms}$, we choose $\Delta t=1 \mathrm{~ms}$. We consider three network topologies.

a. Directed Erdős-Rényi (ER) network: As a standard model of network topology, we consider a network with random directed connections. Each connection $w_{i j}=1$ is added with probability $p_{\text {con }}$, excluding self-connections $(i, i)$. Then, the degree distribution of outgoing as well as incoming connections follows a binomial distribution with average degree $\bar{k}=p_{\text {con }}(N-1) \simeq p_{\text {con }} N$. We require $p_{\text {con }}>\ln (N) / N$ to ensure that the graph is connected [87]. The connectivity matrix $w_{i j}$ is fixed throughout each simulation, such that averaging over simulations with different network realizations results in a quenched average. A cutout from an example graph is shown in Fig. 2(a).

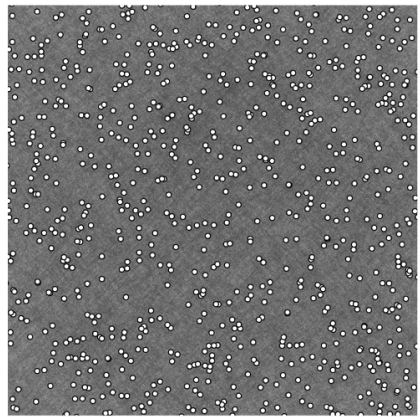

(a)

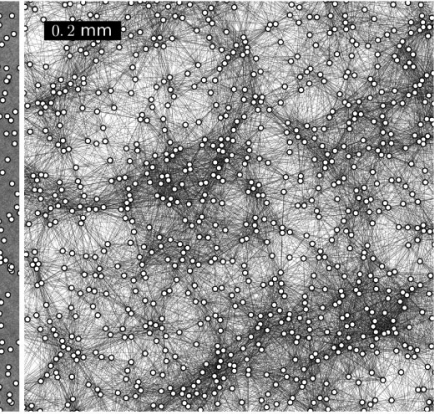

(b)
FIG. 2. Cutouts of two random network topologies. (a) Subset of randomly spaced nodes in an ER network with $p_{\text {con }}=10^{-3}$. Note that connections cross the window also from neurons outside of the field of view, such that single connections cannot be distinguished visually in the sketch. (b) $1.4 \times 1.4 \mathrm{~mm}^{2}$ subset of SC topology generated by axonal-growth rules $[5,88]$. b. Spatially clustered (SC) network: In order to consider a more detailed topology with dominant short-range connections, we follow Orlandi et al., who developed a model based on experimental observations of in vitro cultures $[5,88]$. Neural somata are randomly placed as hard discs with radius $R_{s}=7.5 \mu \mathrm{m}$, to account for the minimal distance between cell centers, on a $5 \times 5-\mathrm{mm}^{2}$ field. From each soma, an axon grows into a random direction with a final length $l$ given by a Rayleigh distribution $p(l)=\left(l / \sigma_{l}^{2}\right) \exp \left(-l^{2} / 2 \sigma_{l}^{2}\right)$, with $\sigma_{l}=900 \mu \mathrm{m}$ and average axonal length $\bar{l} \simeq 1.1 \mathrm{~mm}$. The axonal growth is a semiflexible path with segments of size $\Delta l=10 \mu \mathrm{m}$ and orientation drawn from a Gaussian distribution relative to the previous segment with $\sigma_{\theta}=15^{\circ}$. A connection with another neuron is formed with probability $1 / 2$ if the presynaptic axon intersects with the dendritic tree of a postsynaptic neuron [89]. The dendritic tree is modeled as a disc around the postsynaptic neuron with radius $R_{d}$ drawn from a Gaussian distribution with mean $\bar{R}_{d}=300 \mu \mathrm{m}$ and $\sigma_{d}=20 \mu \mathrm{m}$. A cutout from an example graph is shown in Fig. 2(b).

c. Annealed-average (AA) network: We consider, in addition, a network with $k$ dynamically changing random connections (annealed average). The connections are distinguishable, exclude self-connections, and are redrawn every time step. This model approximates the otherwise numerically expensive fully connected network (ER with $\left.p_{\text {con }}=1\right)$ with a global $m_{t}$ by choosing $\alpha_{j, t}=m_{t} / k$. In practice, we chose $k=4$, which produces analogous dynamics to the fully connected $\left(\bar{k} \approx 10^{4}\right)$ network as long as $m_{t}<4$.

Error bars are obtained as statistical errors from the fluctuations between independent simulations, which include random network realizations $\left\{w_{i j}\right\}$ for ER and SC.

\section{B. Homeostatic plasticity}

In our model, homeostatic plasticity is implemented as a negative feedback, which alters the synaptic strength on the level of the postsynaptic neuron (the homeostatic scaling factor $\alpha_{j, t}$ ) to reach a target neural firing rate $r_{j}^{*}$. We consider a linear negative feedback with time constant $\tau_{\mathrm{hp}}$, which depends solely on the (local) activity of the postsynaptic neuron $s_{j, t}$,

$$
\Delta \alpha_{j, t}=\left(\Delta t r_{j}^{*}-s_{j, t}\right)\left(\frac{\Delta t}{\tau_{\mathrm{hp}}}\right),
$$

i.e., adapting a neuron's synaptic strength does not rely on information about the population activity $A_{t}$. Since $\alpha_{j, t}$ is a probability, we additionally demand $\alpha_{j, t} \geq 0$. Equation (6) considers homeostatic plasticity to directly couple to all postsynaptic synapses of any given neuron $j$. This can be implemented biologically as autonomous synaptic processes or somatic processes, such as translation and 
transcription. In order to further reduce complexity, we assume a uniform target rate $r_{j}^{*}=r^{*}$, while, in fact, experiments show a broad (log-normal) spike-rate distribution [90,91]. Preliminary tests for a log-normal target rate distribution in ER networks ( $p_{\text {con }}=0.1$ ) showed consistent results. In our simulations, we typically consider a biologically motivated target rate $r^{*}=1 \mathrm{~Hz}$ and a homeostatic timescale of the order of an hour, $\tau_{\mathrm{hp}}=10^{3} \mathrm{~s}$.

\section{RESULTS}

Including homeostatic plasticity in our model generates a broad range of dynamic states, depending on the external input. Figure 3 shows qualitatively representative results obtained for AA networks. For strong input $\left[h=\mathcal{O}\left(r^{*}\right)\right]$, the network organizes itself into a dynamic state where neural firing is solely driven by the input fluctuations, resembling an asynchronous-irregular state (green). Here, temporal and pairwise spike count cross-correlations approach zero, and the avalanche-size distribution matches the analytic solution for a Poisson process [92] shown as dashed lines. For weaker input $\left(h<r^{*}\right)$, the system tunes itself towards fluctuating dynamics (orange-yellow). The average neural rate and subsampled avalanche-size distributions are qualitatively similar to reverberant in vivo dynamics with autocorrelation times of several hundred milliseconds (Fig. 1). In this regime, the temporal correlations increase when weakening the input, approaching close-to-critical dynamics, characterized by a power-law distribution $P(s)=s^{-3 / 2}$ [77], at the lower end of the regime. Decreasing the input even further $\left(h \ll r^{*}\right)$ leads to bursting behavior, characterized by silent periods that are interrupted by strong bursts. These bursts are apparent as a peak in the avalanche-size distribution at large avalanche sizes (purple-red). In this regime, the network steadily increases its synaptic strengths during silent periods until a spike initiates a large burst, which, in turn, decreases the synaptic strengths drastically, and so on (cf. Appendix C). This regime captures the qualitative features of bursting in vitro dynamics (Fig. 1).

In the following, we derive a quantitative description of the three regimes sketched above. To quantify the dynamic state, we consider the temporal average of the branching parameter $m=\langle\bar{m}\rangle$, as well as the associated autocorrelation time $\tau$ of the population activity.

\section{A. Mean-field solution}

If we assume that $\tau_{\mathrm{hp}}$ is sufficiently large (i.e., slow homeostatic plasticity), then $\Delta \alpha_{j} \approx 0$ and the dynamics of the network is fully determined by the approximately constant branching parameter $\bar{m}_{t} \approx m$. In this regime, Eq. (4) holds and, combined with Eqs. (5) and (6), we obtain the mean-field solution

$$
m=1-h / r^{*} \quad \text { and } \quad \tau=-\Delta t / \ln \left(1-h / r^{*}\right) .
$$

Hence, with decreasing input rate $h$, recurrent network activation $(m)$ increases; i.e., perturbations cause a stronger network response and the autocorrelation time increases (Fig. 4, solid lines).

In the light of this mean-field solution, we discriminate the three characteristic regimes as follows. First, we define the input-driven regime by $m \leq 0.5$ and $\tau \approx \Delta t$. Here, the network activity is dominated by input $\left[h=\mathcal{O}\left(r^{*}\right)\right]$, and, thus, the dynamics follows the input statistics and becomes irregular. Second, we define the fluctuating regime for $0.5<m<1$ with a nonvanishing but finite autocorrelation
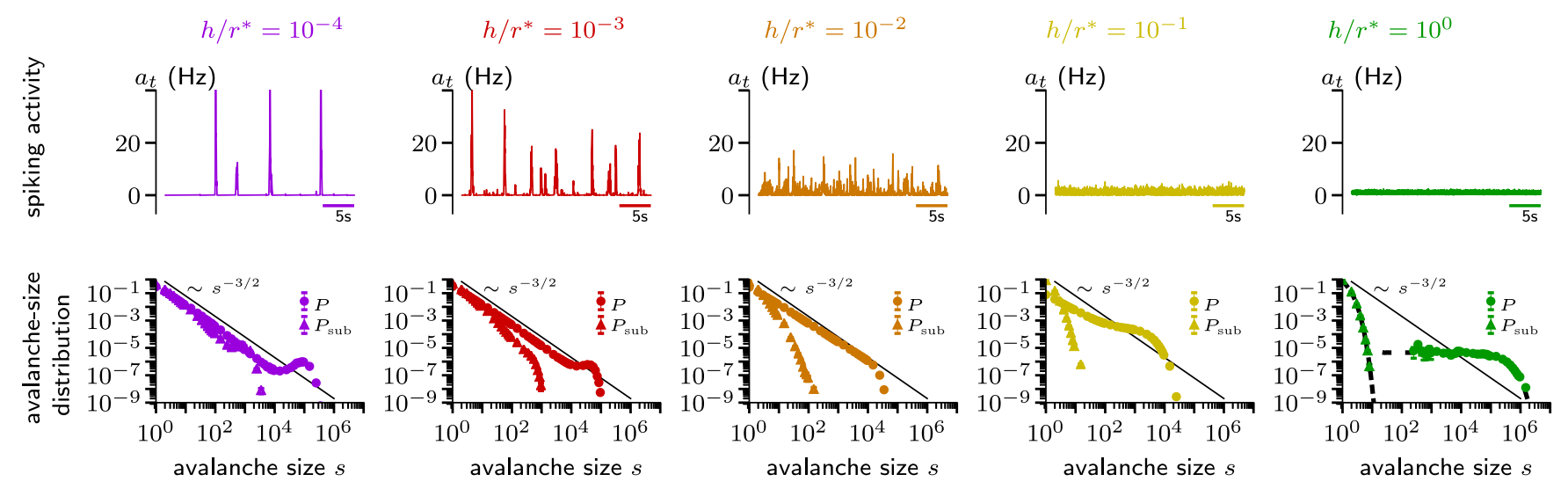

FIG. 3. Homeostatic plasticity induces diverse dynamic states by regulating recurrent network interactions, mediating input rate $h$ and target neural firing rate $r^{*}$. The annealed-average network reproduces bursting $\left(m>1, h / r^{*} \leq 10^{-3}\right.$, purple-red); fluctuating $(m \approx 0.99$, $h / r^{*} \approx 10^{-2}$ and $m \approx 0.9, h / r^{*} \approx 10^{-1}$, orange-yellow); and irregular $\left(m \approx 0, h / r^{*}=1\right.$, green) dynamics. The top row shows examplary spiking activity $a_{t}=A_{t} / N \Delta t$ (Appendix B-1); the bottom row shows avalanche-size distributions $P(s)(n=N$, circles) and subsampled avalanche-size distributions $P_{\text {sub }}(s)(n=100$, triangles) averaged over 12 independent simulations (Appendix B-2). Solid lines show the power-law distribution $P(s) \propto s^{-3 / 2}$ [77], and dashed lines show the analytical avalanche-size distribution of a Poisson process [92]. The parameters are $N=10^{4}, \tau_{\mathrm{hp}}=10^{3} \mathrm{~s}, r^{*}=1 \mathrm{~Hz}, \Delta t=1 \mathrm{~ms}$. 

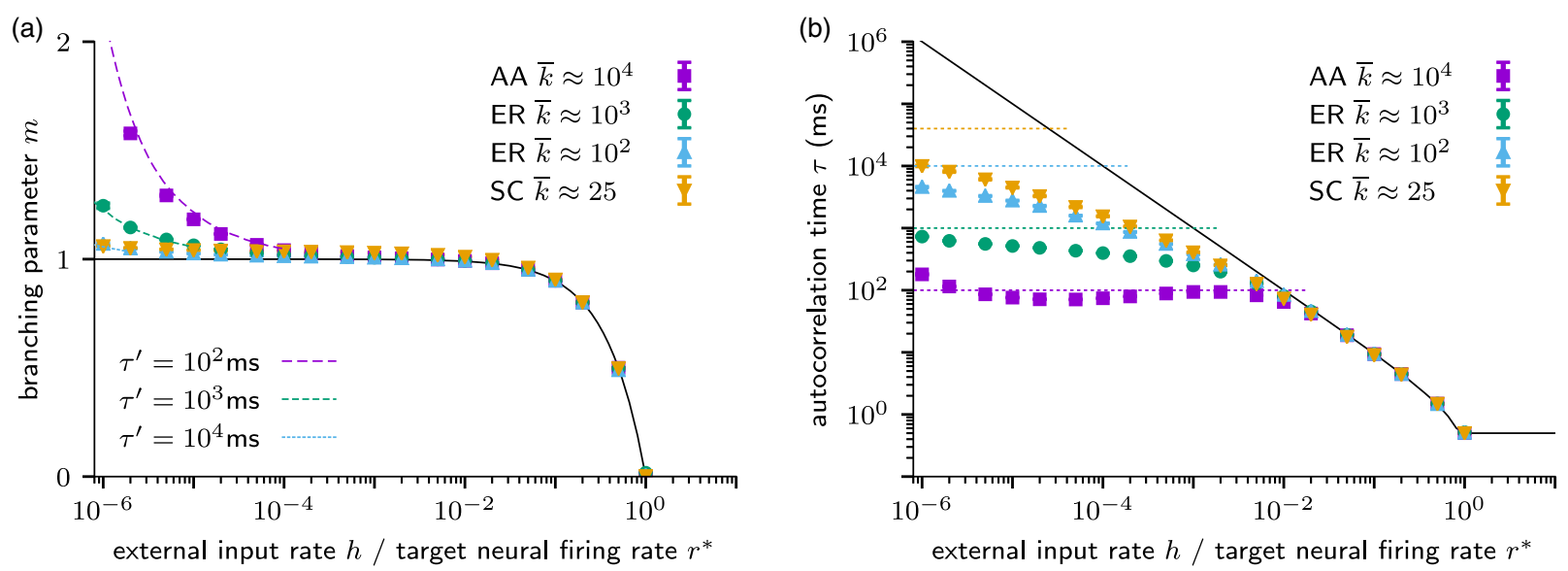

FIG. 4. Quantitative distinction between dynamic states induced in neural networks of different topologies by homeostatic plasticity as a function of normalized input rate $h / r^{*}$. Data points are averages over 12 independent simulations $\left(N=10^{4}, \tau_{\mathrm{hp}}=10^{3} \mathrm{~s}, r^{*}=1 \mathrm{~Hz}\right.$, $\Delta t=10^{-3} \mathrm{~s}$ ) with connections generated according to AA, ER, or SC topologies with average number of connections $\bar{k}$. Solid lines show the mean-field solution in Eq. (7); dashed lines represent (semianalytical) approximations of the bursting regime. (a) Branching parameter $m=\langle\bar{m}\rangle$ varies from irregular $(m \approx 0)$, to fluctuating $(m \lesssim 1)$, to bursting $(m>1)$ dynamics. The behavior in the bursting regime strongly depends on the network timescale $\tau^{\prime}=\tau_{\text {hp }} / \bar{k}$. (b) Integrated autocorrelation time of the network population activity (Appendix B-3) shows a crossover from irregular $[\tau=\mathcal{O}(\Delta t)]$, over fluctuating $\left[\tau=-\Delta t / \ln \left(1-h / r^{*}\right)\right]$ to bursting $\left(\tau \approx \tau^{\prime}\right)$ dynamics.

time $\Delta t<\tau<\infty$. Here, the network maintains and amplifies input as recurrently generated fluctuations. In these two regimes, the mean-field solution in Eq. (7) matches numerical data on different network topologies (Fig. 4). Third, the mean-field solution predicts that, in the limit $h \rightarrow 0$, the dynamics become critical with divergent autocorrelation time $(m \rightarrow 1, \tau \rightarrow \infty)$. However, we observe a clear deviation from the mean-field solution, which defines the bursting regime with $m>1$ and a finite autocorrelation time, as discussed below.

\section{B. Bursting regime}

Deviations from the mean-field solution in Eq. (7) emerge when the assumption of "sufficiently large $\tau_{\mathrm{hp}}$ " breaks down. We will derive a bound for $\tau_{\mathrm{hp}}$, below which the (rapid) homeostatic feedback causes notable changes of the network branching parameter $\bar{m}_{t}$ around its mean $m=\langle\bar{m}\rangle$, which in turn jeopardize the stability of the network dynamics.

To estimate the change of the network branching parameter, we first consider the change in local branching parameter $\Delta m_{i, t}$, which depends on each neuron's outdegree $k_{i}=\sum_{j=1}^{N} w_{i j}$ and is given by

$$
\Delta m_{i, t}=\sum_{j=1}^{N} w_{i j} \Delta \alpha_{j, t}=\left(k_{i} \Delta t r^{*}-\sum_{j=1}^{N} w_{i j} s_{j, t}\right)\left(\frac{\Delta t}{\tau_{\mathrm{hp}}}\right) .
$$

On the network level, we make the assumption that the state of each neuron is approximated by the network average $s_{i, t} \approx A_{t} / N$, such that $\overline{\sum_{j=1}^{N} w_{i j} s_{j, t}} \approx \bar{k} \frac{A_{t}}{N}$. Then, the change in network branching parameter can be approximated as

$$
\begin{aligned}
\Delta \bar{m}_{t} & =\overline{\Delta m}_{t} \approx\left(\bar{k} \Delta t r^{*}-A_{t} \frac{\bar{k}}{N}\right)\left(\frac{\Delta t}{\tau_{\mathrm{hp}}}\right) \\
& \approx\left(\Delta t r^{*}-\frac{A_{t}}{N}\right)\left(\frac{\Delta t}{\tau^{\prime}}\right),
\end{aligned}
$$

where we have introduced an effective homeostatic network timescale $\tau^{\prime}=\tau_{\mathrm{hp}} / \bar{k}$, for which Eq. (8) recovers the form of Eq. (6). Using $\tau^{\prime}$ allows one to semianalytically approximate the deviation of $m$ from the mean-field solution [Fig. 4(a), dashed lines, and Appendix C].

We next show that the stability of network dynamics requires the autocorrelation time of the dynamic process $\tau$ to be smaller than the timescale of homeostasis $\tau^{\prime}$. Stability demands that the homeostatic change in autocorrelation time $\Delta \tau$ is small compared to the autocorrelation time itself, i.e., $\Delta \tau \ll \tau$. We approximate $\Delta \tau$ by error propagation in Eq. (5), yielding

$$
\Delta \tau \simeq\left|\left(\tau^{2} / \Delta t\right) e^{\Delta t / \tau}\right| \Delta m \simeq\left(\tau^{2} / \Delta t+\tau\right) \Delta m,
$$

where we expanded the exponential for small $\Delta t / \tau$. For large $\tau$, the leading term in Eq. (9) dominates, and inserting Eq. (8) yields $\Delta \tau \simeq\left|\Delta t r^{*}-A_{t} / N\right|\left(\tau^{2} / \tau^{\prime}\right)$. Thus, the dynamics can be described as a stationary branching process (mean-field solution) only as long as

$$
\tau \ll \tau^{\prime}\left|\Delta t r^{*}-A_{t} / N\right|^{-1} .
$$

Violation of Eq. (10) results in bursting behavior (Figs. 3 and 7). For $A_{t}=\mathcal{O}(N)$, the right-hand side of Eq. (10) is minimal, because $\Delta t r^{*} \ll 1$, which implies a maximal attainable autocorrelation time $\tau \simeq \tau^{\prime}=\tau_{\mathrm{hp}} / \bar{k}$. This is in perfect agreement with the saturation of measured 
autocorrelation time in the bursting regime [Fig. 4(b), dashed lines].

The transition from the fluctuating to the bursting regime occurs when the mean-field solution in Eq. (7) equals the maximal attainable autocorrelation time, i.e., $\tau=-\Delta t /$ $\ln \left(1-h / r^{*}\right) \approx \tau^{\prime}$. Hence, the transition occurs at $h / r^{*} \approx$ $1-e^{-\Delta t / \tau^{\prime}} \approx \Delta t / \tau^{\prime}$. For an even lower input rate, the dynamics become more and more bursty, and the avalanche-size distribution exhibits a peak at large avalanche sizes (Fig. 3 for $h / r^{*}<10^{-2}$, where $\tau^{\prime}=10^{2} \mathrm{~ms}$, $\Delta t=1 \mathrm{~ms})$. At the transition, the dynamics can be considered close to critical, because the (fully sampled) avalanche-size distribution is closest to a power law with exponent $-3 / 2$.

\section{Distributions of spiking activity}

The different dynamical regimes imply characteristic distributions of neural network activity $P\left(a_{t}\right)$. Figure 5 shows an example of $P\left(a_{t}\right)$ for ER networks with $p_{\text {con }}=10^{-2}$, where the transition from fluctuating to bursting dynamics is expected for $h / r^{*} \approx \Delta t / \tau^{\prime}=10^{-4}$. In the irregular regime (green), $P\left(a_{t}\right)$ is a unimodal distribution. In the fluctuating regime (yellow-red), the peak in $P\left(a_{t}\right)$ shifts towards quiescence, and the distribution develops a power-law tail with exponential cutoff, which is expected for a critical branching process. In the bursting regime (purple-blue), $P\left(a_{t}\right)$ is a bimodal distribution, reflecting network changes between quiescence and bursty activity. The position and sharpness of the high-activity maximum depend on the network connectivity and, hence, the heterogeneity in the single-neuron input.

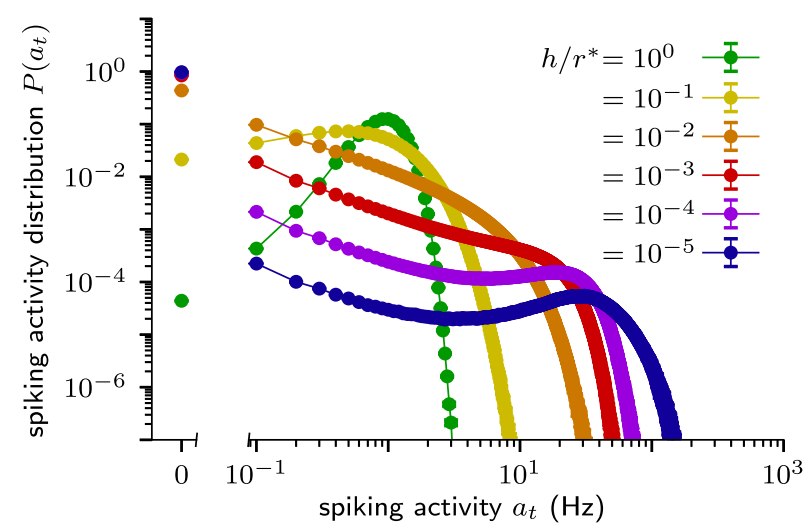

FIG. 5. Distribution of spiking activity in weakly connected Erdős-Rényi networks $\left(p_{\text {con }}=10^{-2}, \Delta t=1 \mathrm{~ms}, r^{*}=1 \mathrm{~Hz}\right.$, $\tau^{\prime}=10^{4} \mathrm{~ms}$ ) averaged over 12 independent simulations. For irregular dynamics $\left(h / r^{*} \approx 10^{0}\right)$, the distribution is clearly unimodal. For fluctuating dynamics $\left(10^{-4}<h / r^{*}<10^{0}\right)$, the distribution broadens and shifts the maximum towards quiescence. In addition, towards the lower bound of the regime, the distribution develops a power-law tail with an exponential cutoff. At the crossover to bursting dynamics $\left(h / r^{*} \approx 10^{-4}\right)$ the distribution becomes bimodal.

\section{Reproducing experimental results}

Using the insight from our theory, we can reproduce experimental results. Spiking activity recorded in vivo resembles dynamics of the fluctuating regime. In this regime, the dynamic state is consistent for all topologies we considered (Fig. 4). Therefore, already a branching network on an AA topology suffices to quantitatively reproduce the avalanche-size distributions by matching model parameters with experimentally accessible estimates (Fig. 1, dashed lines). To match the branching network to recordings from cat $\mathrm{V} 1$ and rat $\mathrm{CA} 1$, we first estimated the spike rate $r$ and autocorrelation time $\tau$ from the recordings of spiking activity [80]; we then chose biologically plausible parameters for the network size $N$, the homeostatic timescale $\tau_{\mathrm{hp}}$, as well as the simulation time step $\Delta t$; and finally, we derived the external input $h$ using Eq. (7) (for details, see Appendix B-4). The resulting subsampled avalanche-size distributions are in astonishing agreement with the experimental results, given the simplicity of our approach. Close inspection of the avalanche-size distribution for rat CA1 recordings still reveals small deviations from our model results. The deviations can be attributed to theta oscillations in the hippocampus, which result in subleading oscillations on an exponentially decaying autocorrelation function [80]. While this justifies our approach to consider a single dominant autocorrelation time, theta oscillations slightly decorrelate the activity at short times and thereby foster premature termination of avalanches. Thus, the tail in the avalanche-size distribution is slightly shifted to smaller avalanche sizes (Fig. 1).

The in vitro results are qualitatively well matched by simulations in the bursting regime, with avalanche-size distributions showing a characteristic peak at large avalanche sizes (Fig. 3). It is difficult to quantitatively match a model to the data, because a number of parameters can only be assessed imprecisely. Most importantly, the autocorrelation time in the burst regime is not informative about the external input rate $h$ and depends on the average number of connections (Fig. 4). Likewise, the time dependence of the branching parameter $m_{t}$ cannot be assessed directly. Finally, system size and topology impact the network dynamics more strongly in this regime than in the fluctuating or input-driven regime. This yields a family of avalanche-size distributions with similar qualitative characteristics but differences in precise location and shape of the peak at large sizes.

\section{DISCUSSION}

We propose the interplay of external input rate and target spike rate, mediated by homeostatic plasticity, as a neural mechanism for self-organization into different dynamic states (cf. sketch in Fig. 6). Using the framework of a branching process, we disentangled the recurrent network dynamics from the external input (e.g., input from other brain areas, external stimuli, and spontaneous spiking of individual neurons). Our mean-field solutions, 


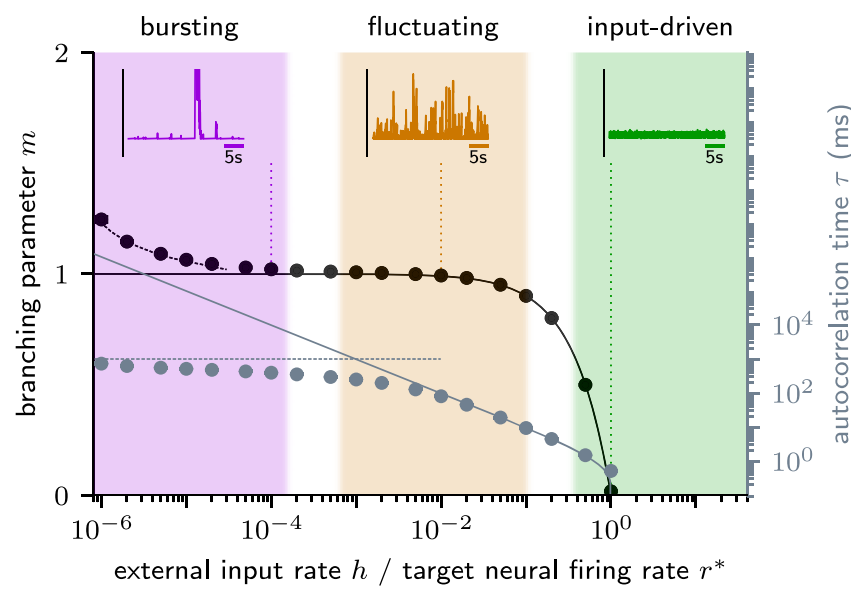

FIG. 6. Sketch of bursting, fluctuating, and input-driven network states, classified by the branching parameter and the autocorrelation time. We propose (solid lines) that homeostatic plasticity tunes the dynamic state depending on the ratio of external input rate (including spontaneous neural firing) and target neural firing rate. Data points and example activity traces stem from Erdős-Rényi networks $\left(N=10^{4}, \quad p=10^{-1}\right.$, $\left.\tau_{\mathrm{hp}}=10^{3} \mathrm{~s}\right)$. In the bursting regime, the homeostatic timescale $\tau_{\mathrm{hp}}$ influences the resulting dynamics (dashed lines).

complemented by numeric results for generic spiking neural networks, show that, for high input, the network organizes into an input-driven state, while for decreasing input, the recurrent interactions are strengthened, leading to a regime of fluctuating dynamics, resembling the reverberating dynamics observed in vivo. Decreasing the input further induces bursting behavior, known from in vitro recordings, due to a competition of timescales between homeostatic plasticity and the autocorrelation of population activity. Thereby, our framework proposes a generic mechanism to explain the prominent differences between in vivo and in vitro dynamics.

Our theory suggests that also differences within the collective dynamic state observed in vivo can be explained by considering differences in input strength. For the cortex, it was shown that layer $2 / 3$ exhibits critical dynamics [17] and, presumably, deeper layers show reverberating dynamics $[80,81]$. We propose that this can be caused by different input strengths: Layer $2 / 3$ is more recurrently connected, while layer 4 is the main target of thalamic input [93], hence receiving the stronger input. The dynamic state varies also across different cortical areas, where autocorrelation times of network activity reflect a hierarchical organization [8,94]: Cortical areas associated with higher-order function show a larger autocorrelation time. In light of our results, a larger autocorrelation time implies less afferent input for the area in question. The hierarchical organization is further supported by our analysis of spiking activity in vivo (Fig. 1): The autocorrelation times in the visual cortex $(\tau \approx 0.2 \mathrm{~s})$ and hippocampus $(\tau \approx 2 \mathrm{~s})$ precisely reflect that the visual cortex is almost at the bottom, whereas the hippocampus is at the top of the hierarchy of visual processing [95].

Our theory provides an approach to experimentally infer the fraction of spikes generated recurrently within a network and generated by external input. For an average spike rate $r$, Eq. (7) implies $h / r=\left(1-e^{-\Delta t / \tau}\right)$. The external input rate can then be directly calculated from the autocorrelation time and by assuming a biologically plausible signal-propagation time, e.g., $\Delta t \approx 4 \mathrm{~ms}$. We estimate for recordings from the visual cortex in a mildly anesthetized cat that about $2 \%$ of the network activity is generated by the input, whereas the majority of $98 \%$ is generated recurrently within the network. From autocorrelation times measured across the cortical hierarchy $(50-350 \mathrm{~ms})$ in a macaque monkey [8], the fraction of spikes generated by external input decreases from approximately $8 \%$ to $1 \%$ from lower to higher cortical areas. This is consistent with perturbation experiments in rat barrel cortex, where, after triggering an extra spike, the decay time of the population rate was at least $50 \mathrm{~ms}$ [96], indicating at most about 8\% external input (for a detailed discussion, see also Ref. [97]). Last, experiments on the visual cortex of awake mice directly after thalamic silencing found a decay time of $\tau=12(1) \mathrm{ms}$ [98], from which we would estimate about $70 \%$ recurrent activation. This is in perfect agreement with the experimentally measured $72(6) \%$ of recurrent activation in the same study. This result thus validates our derived relation between $h / r$ and $\tau$.

One can interpret our findings in the light of up and down states $[18,19,67,99]$. Because the membrane potential was found to correlate with network activity $[6,20]$, our results for the distribution of spiking activity in the bursting regime may correspond to the bimodal distributions of membrane potentials during up and down states (Fig. 5). It has already been shown that negative feedback can stabilize up and down states $[67,99]$. In our theory, negative feedback leads to similar results in the low-input regime. Moreover, we predict that decreasing network input further prolongs the quiescent periods or down states.

Our theory unifies previous numerical approaches of selforganization in neural networks, which typically considered a negative feedback mechanism but made very different choices on a (fixed) network input. For example, bursting dynamics have been generated by homeostatic buildup upon loss of network input [45] or by self-organized supercriticality through dynamic neuronal gain [100]. Adding weak input, self-organized criticality [101,102] has been achieved by local rewiring [57-59] and synaptic depression [53,54,60-65]. In contrast, asynchronousirregular network activity typically requires a comparably strong input, assuming a balanced state $[49,103,104]$, and a self-organized AI network state can be promoted by inhibitory plasticity $[55,56]$. While all these studies provide mechanisms of self-organization to one particular dynamic state, our theory highlights the role of input in combination 
with a negative feedback [42,49-51] and provides a unifying mechanism of self-organization covering bursting, fluctuating, and irregular dynamics.

From a broader perspective, we characterized driven systems with a negative feedback as a function of the input rate. The negative feedback compensates the input by regulating the system's self-activation to achieve a target activity. In light of this control theory, the bursting regime can be understood as resonances in a feedback loop, where feedback dynamics are faster than system dynamics (cf. Ref. [105]). This qualitative picture should remain valid for other connected graphs subject to external input with spatial and temporal correlations. In this case, however, we expect more complex network responses than predicted by our mean-field theory, which assumes selfaveraging random networks subject to uncorrelated input.

Our results suggest that homeostatic plasticity may be exploited in experiments to generate in vivo-like dynamics in a controlled in vitro setup, in particular to abolish the ubiquitous bursts in vitro. Previous attempts to reduce bursting in vitro [106] and in model systems of epilepsy [107-110] used short-term electrical and optical stimulation to attain temporal reduction in bursting. Alternatively, one can reduce bursting pharmacologically or by changing the calcium level, however, typically at the cost of changing single-neuron properties [111-113]. We propose a different approach, namely, applying weak, global, long-term stimulation. Mediated by homeostasis, the stimulation should alter the effective synaptic strength, and thereby the dynamic state, while preserving single-neuron dynamics [114]. In particular, we predict that inducing in every neuron additional spikes with $h=\mathcal{O}(0.01 \mathrm{~Hz})$ is sufficient to abolish the ubiquitous bursts in vitro and render the dynamics in vivo-like instead. If verified, this approach promises completely novel paths for drug studies. By establishing in vivo-like dynamics in vitro, fine differences between neurological disorders, which are otherwise masked by the ubiquitous bursts, can be readily identified. Altogether this would present a comparably cost-efficient, high-throughput, and highly accessible drug assay with largely increased sensitivity.

\section{ACKNOWLEDGMENTS}

We would like to thank Manuel Schottdorf and Andreas Neef, as well as Raoul-Martin Memmesheimer and Sven Goedeke, for stimulating discussions. We are grateful for careful proofreading from Roman Engelhardt, João Pinheiro Neto, and Conor Heins. All authors acknowledge funding by the Max Planck Society. J.Z. and V.P. received financial support from the German Ministry of Education and Research (BMBF) via the Bernstein Center for Computational Neuroscience (BCCN) Göttingen under Grant No. 01GQ1005B. J.W. was financially supported by Gertrud-ReemtsmaStiftung and Physics-to-Medicine Initiative Göttingen (ZN3108) LM des Niedersächsischen Vorab.

\section{APPENDIX A: EXPERIMENTAL DETAILS}

1. Dissociated dense cultures of cortical rat neurons.The spike-time data from dissociated cortical rat neurons of mature dense cultures was recorded by Wagenaar et al. [4] and was obtained freely online [74]. The experimental setup uses multielectrode arrays (MEA) with $n=59$ electrodes. Cortical cells were obtained from dissecting the anterior part of the cortex of Wistar rat embryos (E18), including somatosensory, motor, and association areas. For details, we refer to Ref. [4]. Measurements were performed every day in vitro (DIV). We here focus on the dense case with 50000 cells plated initially with a density of $2.5(1.5) \times 10^{3}$ cells $/ \mathrm{mm}^{2}$ at $1 \mathrm{DIV}$, which is compatible with standard in vitro experiments in the field that claim to observe critical dynamic behavior. We selected the representative recordings 8-2-34 (exp 1) and 7-2-35 (exp 2) at a mature age (34/35 DIVs) for Fig. 1.

2. Rat hippocampus.-The spiking data from rats were recorded by Mizuseki et al. [21,75] with experimental protocols approved by the Institutional Animal Care and Use Committee of Rutgers University. The data were obtained from the NSF-founded CRCNS data sharing website [75]. The spikes were recorded in CA1 of the right dorsal hippocampus during an open field task. Specifically, we used the data set ec013.527 with sorted spikes from 4 shanks with $n=31$ channels. For details, we refer to Refs. [21,75].

3. Primary visual cat cortex.-The spiking data from cats were recorded by Tim Blanche in the laboratory of Nicholas Swindale, University of British Columbia, in accordance with guidelines established by the Canadian Council for Animal Care [22,76]. The data were obtained from the NSFfounded CRCNS data sharing website [22]. Specifically, we used the data set pvc3 with recordings of $n=50$ sorted single units [76] in area 18. For details, we refer to Refs. [22,76]. We confined ourselves to the experiments where no stimuli were presented, such that spikes reflect the spontaneous activity in the visual cortex of mildly anesthetized cats. In order to circumvent potential nonstationarities at the beginning and end of the recording, we omitted the initial $25 \mathrm{~s}$ and stopped after $320 \mathrm{~s}$ of recording [80].

\section{APPENDIX B: ANALYSIS DETAILS}

1. Spiking activity.-In order to present the spiking activity over time, we partition the time axis of experimental or numerical data into discrete bins of size $\Delta t$. For the time-discrete simulations, the time bin naturally matches the time step. For experimental data, we set $\Delta t=4 \mathrm{~ms}$. In each time bin, we count the total number of spikes $A_{t}$ and normalize with the number of neurons $N$ to obtain the average spiking activity $a_{t}=A_{t} / N \Delta t$. Note that experimental preparations were inevitably subsampled, as spikes were recorded only from a small number of all neurons. 
2. Avalanche-size distribution.-We define the avalanche size $s$ as the number of spikes enclosed along the discrete time axis by bins with zero activity [7]. To test for criticality in terms of a branching process, one compares $P(s)$ to the expected $P(s) \sim s^{-3 / 2}$. This is a valid approach in the limit $h \rightarrow 0$, where avalanches can be clearly identified, and for fully sampled systems [81]. However, experiments are limited to record only from $n$ out of $N$ neurons. As a result, the distributions for subsampled activity $P_{\text {sub }}(s)$ differ due to subsampling bias $[15,16]$. Therefore, we numerically measure both full $(n=N)$ and subsampled $(n<N)$ avalanchesize distributions to qualitatively compare $P(s)$ to the theory and $P_{\text {sub }}(s)$ to experimental data.

3. Integrated autocorrelation time.-We measure the autocorrelation time of spiking activity $a_{t}$ in terms of the integrated autocorrelation time $\tau_{\text {int }}$; for details, see, e.g., Ref. [115]. In brief, we sum over the normalized autocorrelation function $C(l)=\operatorname{Cov}\left[a_{t}, a_{t+l}\right] / \operatorname{Var}\left[a_{t}\right]$ until the sum converges. Following conventions, we define $\tau_{\text {int }}=$ $\Delta t\left[\frac{1}{2}+\sum_{l=1}^{l_{\max }} C(l)\right]$, where $l_{\max }$ is self-consistently obtained as the minimal $l_{\max }>6 \tau_{\text {int }}\left(l_{\max }\right)$.

4. Reproducing experimental results.-We use a branching network with AA topology subject to homeostatic plasticity to quantitatively reproduce in vivo subsampled avalanche-size distributions. We chose networks of size $N=10^{4}$, with sufficiently large homeostatic timescale $\tau_{\mathrm{hp}}=10^{5} \mathrm{~s}$. The following model parameters can be obtained from experimentally measured values: In the chosen recordings, we measured the average rate ( $r_{\text {cat }} \approx 7 \mathrm{~Hz}$ and $r_{\text {rat }} \approx 11 \mathrm{~Hz}$ ) as well as the subsampling corrected branching parameter [80] $\left(m_{\mathrm{cat}} \approx 0.98\right.$ and $m_{\mathrm{rat}} \approx$ 0.997 for $\Delta t=4 \mathrm{~ms}$ ). In fact, the branching parameter is not suitable to identify the input rate via Eq. (7), because it refers to a process in discrete time steps. Since we are treating a continuous process, the invariant quantity is the autocorrelation time ( $\tau_{\text {cat }} \approx 0.2 \mathrm{~s}$ and $\tau_{\text {rat }} \approx 1.6 \mathrm{~s}$ ). According to our theory, we can then calculate the input rate per neuron $h=[1-\exp (-\Delta t / \tau)] r$. In order to avoid convergence effects, we need to choose a sufficiently small time step $\Delta t=1 \mathrm{~ms}$ of signal propagation (resulting in $h_{\text {cat }} \approx 3.5 \times 10^{-2} \mathrm{~Hz}$ and $h_{\text {rat }} \approx 5.5 \times 10^{-3} \mathrm{~Hz}$ ), while we record in time bins of $4 \mathrm{~ms}$ to match the analysis of the experiments. Subsampled avalanche-size distributions are estimated by randomly choosing $n<N$ neurons, where we approximated $n$ by the number of electrodes or channels $\left(n_{\text {cat }}=50\right.$ and $\left.n_{\text {rat }}=31\right)$.

\section{APPENDIX C: APPROXIMATING THE DYNAMIC STATE IN THE BURSTING REGIME}

We showed in Sec. IV that decreasing the external input to recurrent networks with homeostatic plasticity leads to bursting behavior [Fig. 7(a)]. This is directly related to the network branching parameter $m_{t}=\bar{m}_{t}$ no longer showing small fluctuations around the predicted value but instead

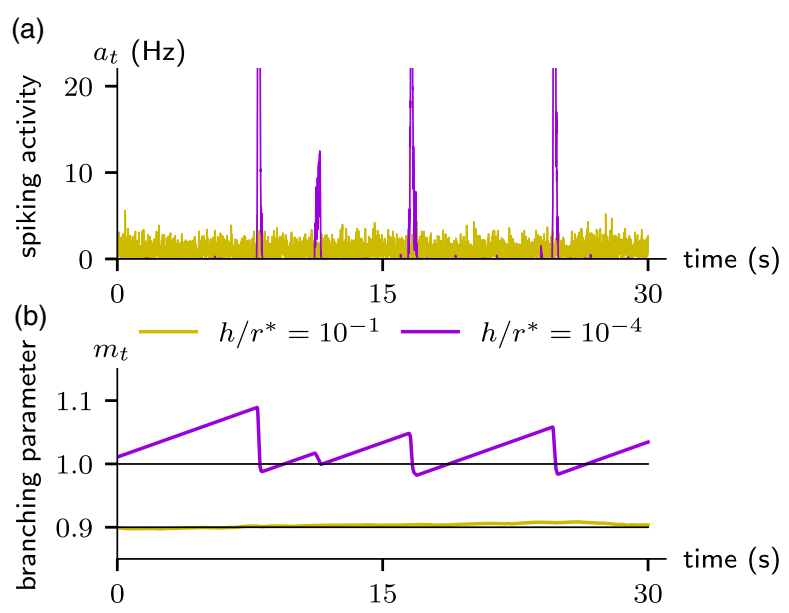

FIG. 7. Temporal fluctuations in an annealed-average network with homeostatic plasticity subject to different external input rates. (a) Spiking activity $a_{t}$ shows small fluctuations for large input rates (yellow) and bursts for small input rates (purple); cf. Fig. 3. (b) Branching parameter fluctuates around predicted value (black horizontal lines) and develops a distinct sawtooth pattern for small input rates.

exhibiting a prominent sawtooth pattern [Fig. 7(b)], a hallmark of the homeostatic buildup in the long pauses with no input.

We here show a semianalytical approximation of the network branching parameter in the bursting regime. For sufficiently small external input, we may assume separation of timescales; i.e., every externally induced spike drives one avalanche with periods of silence in between. Let us first consider the periods of silence, i.e., no activity per site. This holds during the entire growth period $T$ such that Eq. (8) yields

$$
m_{t}-m_{t-T}=\left(\Delta t r^{*}\right) \frac{T}{\tau^{\prime}}
$$

The situation becomes more involved within the bursts, where the behavior of $m_{t}$ is nonlinear. Consider an external spike that triggers an avalanche at $t=s$, which ends at $t=e$. Because of the separation of timescales, we can assume $A_{s}=1$. There are two possible scenarios: (i) The avalanche dies out before a burst can develop and (ii) the input triggers a proper burst with a macroscopic activation.

We first estimate the probability that an avalanche dies out before a burst develops. For $\tau_{\text {hp }} \gg \Delta t$, we approximate $m_{t} \approx m_{s}=$ const. Then, the probability of ultimate extinction $\theta$ can be calculated as the solution of $\theta=\Pi(\theta)$ with $\Pi(\theta)$ the probability generating function [77]. In the onset phase, the branching process is described by a Poisson process per event with mean $m_{s}$, such that $\Pi(\theta)=e^{-m_{s}(1-\theta)}$. We are, thus, looking for a solution of

$$
\theta=e^{-m_{s}(1-\theta)},
$$


which can be rewritten to

$$
-m_{s} \theta e^{-m_{s} \theta}=-m_{s} e^{-m_{s}} .
$$

We identify the Lambert-W function $W(z) e^{W(z)}=z$ [116] with $W(z)=-m_{s} \theta=W\left(-m_{s} e^{-m_{s}}\right)$ and find for the probability that no burst develops

$$
p_{\text {no-burst }}\left(m_{s}\right)=\theta=-\frac{1}{m_{s}} W\left(-m_{s} e^{-m_{s}}\right) .
$$

If a proper burst develops, the strong activity diminishes $m_{t}$ until the burst dies out again. We cannot analytically estimate the branching parameter $m_{e}$ after burst end, but we can use a deterministic numerical approximation to obtain $m_{e}\left(m_{s}\right)$. Instead of stochastically generating new (discrete) events according to some distribution $P\left(m_{t}\right)$ with average $m_{t}$, we approximate the branching process as deterministic (continuous) evolution $A_{t+1}=m_{t} A_{t}$. For a finite network, we need to consider convergence effects when one neuron is activated by two or more neurons at the same time. In the absence of external input, this introduces for an AA (i.e., approximating fully connected) network the activitydependent branching parameter [117]

$$
m_{t}\left(A_{t}\right)=\frac{N}{A_{t}}\left(1-\left(1-\frac{m_{t}}{N}\right)^{A_{t}}\right)
$$

which we need to consider for the activity propagation within the burst, i.e., $A_{t+1}=m_{t}\left(A_{t}\right) A_{t}$. In addition, we introduce an upper bound $A_{t} \leq N$. The upper limit on $A_{t}$ puts a lower bound on $\Delta m_{t}$, according to Eq. (8), and thus, extends the duration of avalanches. Evolving $m_{t+1}=m_{t}+\left(\Delta t r^{*}-A_{t} / N\right)\left(\Delta t / \tau^{\prime}\right)$, with $m_{t+1} \geq 0$, we iterate until $A_{e}<1$. This is a quick and numerically robust iterative scheme to estimate $m_{e}\left(m_{s}\right)$.

Putting everything together, we numerically approximate the average network branching parameter $m$ under homeostatic plasticity in the bursting regime of low external input for an AA network. For this, we sample the external spikes (drive) as $10^{4}$ interdrive intervals $T_{s}$ from an exponential distribution $P(T)=(1 / h N) e^{-T / h N}$, corresponding to $N$ Poisson processes with rate $h$. The remaining part can be interpreted as an event-based sampling with approximate transformations: Starting with $m_{0}=0$, we evolve $m_{t}$ for each interdrive interval $T_{s}$ according to Eq. (C1). If $m_{t}>1$, we keep $m_{t}$ with probability $p_{\text {no-burst }}\left(m_{t}\right)$ or else initiate a burst by setting $m_{t}=m_{e}\left(m_{t}\right)$. Afterwards, we continue evolving $m_{t}$.

\section{APPENDIX D: CHARACTERISTIC DURATION OF INTER-BURST-INTERVALS IN BURST REGIME}

In the bursting regime of low external input, the spiking activity suggests a characteristic time between bursts. In order to test for periodicity, we analyzed the distribution of

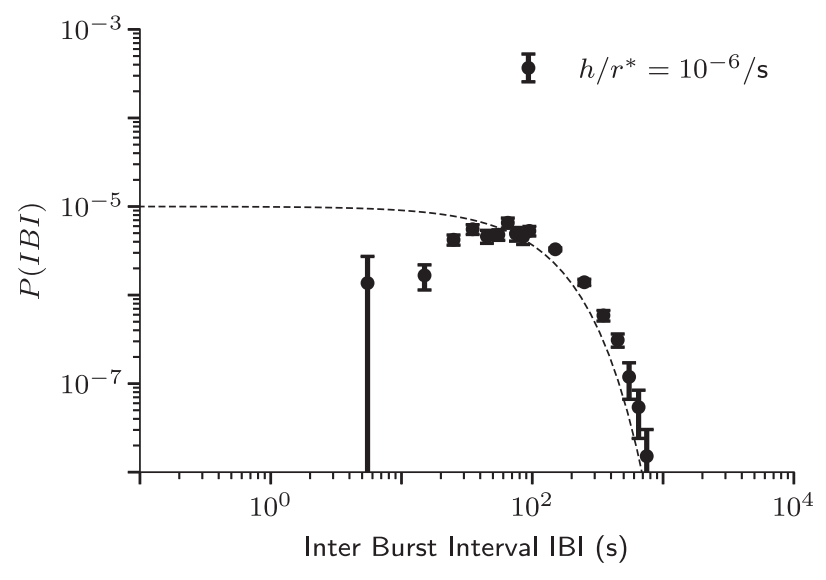

FIG. 8. Inter-burst-interval (IBI) distribution for annealedaverage networks averaged over 12 independent simulations. Intervals are measured as times between proper burst onsets $\left(a_{t}>20 r^{*}\right)$. Dashed lines show the exponential inter-spikeinterval distribution of the Poisson external drive.

interburst intervals (IBI), where intervals are measured as the time between two consecutive burst onsets, defined as a spiking activity $a_{t}>20 r^{*}$. We find (Fig. 8) that large IBI are suppressed by the exponentially distributed interdrive intervals (dashed lines), while short IBI are suppressed by the probability $p_{\text {no-burst }}(m)$ that a given external spike does not trigger a proper burst (Appendix C). This gives rise to a characteristic duration of interburst intervals in the burst regime, although the dynamics are not strictly periodic.

[1] H. P. Robinson, M. Kawahara, Y. Jimbo, K. Torimitsu, Y. Kuroda, and A. Kawana, Periodic Synchronized Bursting and Intracellular Calcium Transients Elicited by Low Magnesium in Cultured Cortical Neurons, J. Neurophysiol. 70, 1606 (1993).

[2] J. Van Pelt, M. A. Corner, P. S. Wolters, W. L. C. Rutten, and G. J. A. Ramakers, Longterm Stability and Developmental Changes in Spontaneous Network Burst Firing Patterns in Dissociated Rat Cerebral Cortex Cell Cultures on Multielectrode Arrays, Neurosci. Lett. 361, 86 (2004).

[3] M. Chiappalone, M. Bove, A. Vato, M. Tedesco, and S. Martinoia, Dissociated Cortical Networks Show Spontaneously Correlated Activity Patterns During In Vitro Development, Brain Res. 1093, 41 (2006).

[4] D. A. Wagenaar, J. Pine, and S. M. Potter, An Extremely Rich Repertoire of Bursting Patterns During the Development of Cortical Cultures, BMC Neurosci. 7, 11 (2006).

[5] J. G. Orlandi, J. Soriano, E. Alvarez-Lacalle, S. Teller, and J. Casademunt, Noise Focusing and the Emergence of Coherent Activity in Neuronal Cultures, Nat. Phys. 9, 582 (2013).

[6] R. Vardi, A. Goldental, S. Sardi, A. Sheinin, and I. Kanter, Simultaneous Multi-Patch-Clamp and Extracellular-Array Recordings: Single Neuron Reflects Network Activity, Sci. Rep. 6, 36228 (2016). 
[7] J. M. Beggs and D. Plenz, Neuronal Avalanches in Neocortical Circuits, J. Neurosci. 23, 11167 (2003).

[8] J. D. Murray, A. Bernacchia, D. J. Freedman, R. Romo, J. D. Wallis, X. Cai, C. Padoa-Schioppa, T. Pasternak, H. Seo, D. Lee, and X.-J. Wang, A Hierarchy of Intrinsic Timescales across Primate Cortex, Nat. Neurosci. 17, 1661 (2014).

[9] B. D. Burns and A. C. Webb, The Spontaneous Activity of Neurones in the Cat's Cerebral Cortex, Proc. R. Soc. B 194, 211 (1976).

[10] W. R. Softky and C. Koch, The Highly Irregular Firing of Cortical Cells Is Inconsistent with Temporal Integration of Random EPSPs, J. Neurosci. 13, 334 (1993).

[11] C. M. Gray, P. König, A. K. Engel, and W. Singer, Oscillatory Responses in Cat Visual Cortex Exhibit Inter-columnar Synchronization Which Reflects Global Stimulus Properties, Nature (London) 338, 334 (1989).

[12] C. M. Gray, Synchronous Oscillations in Neuronal Systems: Mechanisms and Functions, J. Comput. Neurosci. 1, 11 (1994).

[13] G. Buzsáki and A. Draguhn, Neuronal Oscillations in Cortical Networks, Science 304, 1926 (2004).

[14] M. Breakspear, Dynamic Models of Large-Scale Brain Activity, Nat. Neurosci. 20, 340 (2017).

[15] V. Priesemann, M. Munk, and M. Wibral, Subsampling Effects in Neuronal Avalanche Distributions Recorded In Vivo, BMC Neurosci. 10, 40 (2009).

[16] V. Priesemann, M. Valderrama, M. Wibral, and M. Le Van Quyen, Neuronal Avalanches Differ from Wakefulness to Deep Sleep -Evidence from Intracranial Depth Recordings in Humans, PLoS Comput. Biol. 9, e1002985 (2013).

[17] T. Bellay, A. Klaus, S. Seshadri, and D. Plenz, Irregular Spiking of Pyramidal Neurons Organizes as ScaleInvariant Neuronal Avalanches in the Awake State, eLife 4, e07224 (2015).

[18] C. Wilson, Up and Down States, Scholarpedia J. 3, 1410 (2008).

[19] E. A. Stern, A. E. Kincaid, and C. J. Wilson, Spontaneous Subthreshold Membrane Potential Fluctuations and Action Potential Variability of Rat Corticostriatal and Striatal Neurons In Vivo, J. Neurophysiol. 77, 1697 (1997).

[20] R. Cossart, D. Aronov, and R. Yuste, Attractor Dynamics of Network UP States in the Neocortex, Nature (London) 423, 283 (2003).

[21] K. Mizuseki, A. Sirota, E. Pastalkova, and G. Buzsáki, Theta Oscillations Provide Temporal Windows for Local Circuit Computation in the Entorhinal-Hippocampal Loop, Neuron 64, 267 (2009).

[22] T. Blanche, Multi-neuron Recordings in Primary Visual Cortex, CRCNS.org, 2009.

[23] G. G. Turrigiano, K. R. Leslie, N.S. Desai, L. C. Rutherford, and S. B. Nelson, Activity-Dependent Scaling of Quantal Amplitude in Neocortical Neurons, Nature (London) 391, 892 (1998).

[24] D. V. Lissen, S. N. Gomperts, R. C. Carroll, C. W. Christine, D. Kalman, M. Kitamura, S. Hardy, R. A. Nicoll, R. C. Malenka, and M. von Zastrow, Activity Differentially Regulates the Surface Expression of Synaptic AMPA and NMDA Glutamate Receptors, Proc. Natl. Acad. Sci. U.S.A. 95, 7097 (1998).
[25] R. J. O’Brien, S. Kamboj, M. D. Ehlers, K. R. Rosen, G. D. Fischbach, and R. L. Huganir, Activity-Dependent Modulation of Synaptic AMPA Receptor Accumulation, Neuron 21, 1067 (1998).

[26] G. G. Turrigiano and S. B. Nelson, Homeostatic Plasticity in the Developing Nervous System, Nat. Rev. Neurosci. 5, 97 (2004).

[27] G. W. Davis, Homeostatic Control of Neural Activity: From Phenomenology to Molecular Design, Annu. Rev. Neurosci. 29, 307 (2006).

[28] A. H. Williams, T. O'Leary, and E. Marder, Homeostatic Regulation of Neuronal Excitability, Scholarpedia 8, 1656 (2013).

[29] H. Markram and M. Tsodyks, Redistribution of Synaptic Efficacy between Neocortical Pyramidal Neurons, Nature (London) 382, 807 (1996).

[30] M. V. Tsodyks and H. Markram, The Neural Code between Neocortical Pyramidal Neurons Depends on Neurotransmitter Release Probability, Proc. Natl. Acad. Sci. U.S.A. 94, 719 (1997).

[31] M.-f. Fong, J. P. Newman, S. M. Potter, and P. Wenner, Upward Synaptic Scaling Is Dependent on Neurotransmission Rather than Spiking, Nat. Commun. 6, 6339 (2015).

[32] K. Pozo and Y. Goda, Unraveling Mechanisms of Homeostatic Synaptic Plasticity, Neuron 66, 337 (2010).

[33] M. De Pittà, N. Brunel, and A. Volterra, Astrocytes: Orchestrating Synaptic Plasticity?, Neuroscience (N.Y.) 323, 43 (2016).

[34] Y.S. Virkar, W. L. Shew, J. G. Restrepo, and E. Ott, Feedback Control Stabilization of Critical Dynamics via Resource Transport on Multilayer Networks: How Glia Enable Learning Dynamics in the Brain, Phys. Rev. E 94, 042310 (2016).

[35] E. L. Bienenstock, L. N. Cooper, and P. W. Munro, Theory for the Development of Neuron Selectivity: Orientation Specificity and Binocular Interaction in Visual Cortex, J. Neurosci. 2, 32 (1982).

[36] K. D. Miller and D. J. C. MacKay, The Role of Constraints in Hebbian Learning, Neural Comput. 6, 100 (1994).

[37] L. F. Abbott and S. B. Nelson, Synaptic Plasticity: Taming the Beast, Nat. Neurosci. 3, 1178 (2000).

[38] G. G. Turrigiano and S. B. Nelson, Hebb and Homeostasis in Neuronal Plasticity, Curr. Opin. Neurobiol. 10, 358 (2000).

[39] F. Zenke, G. Hennequin, and W. Gerstner, Synaptic Plasticity in Neural Networks Needs Homeostasis with a Fast Rate Detector, PLoS Comput. Biol. 9, e1003330 (2013).

[40] T. Keck et al., Integrating Hebbian and Homeostatic Plasticity: The Current State of the Field and Future Research Directions, Phil. Trans. R. Soc. B 372, 20160158 (2017).

[41] F. Zenke, W. Gerstner, and S. Ganguli, The Temporal Paradox of Hebbian Learning and Homeostatic Plasticity, Curr. Opin. Neurobiol. 43, 166 (2017).

[42] J. Naudé, B. Cessac, H. Berry, and B. Delord, Effects of Cellular Homeostatic Intrinsic Plasticity on Dynamical and Computational Properties of Biological Recurrent Neural Networks, J. Neurosci. 33, 15032 (2013). 
[43] J. Gjorgjieva, J. F. Evers, and S. J. Eglen, Homeostatic Activity-Dependent Tuning of Recurrent Networks for Robust Propagation of Activity, J. Neurosci. 36, 3722 (2016).

[44] P. J. Hellyer, B. Jachs, C. Clopath, and R. Leech, Local Inhibitory Plasticity Tunes Macroscopic Brain Dynamics and Allows the Emergence of Functional Brain Networks, NeuroImage 124, 85 (2016).

[45] F. Fröhlich, M. Bazhenov, and T. J. Sejnowski, Pathological Effect of Homeostatic Synaptic Scaling on Network Dynamics in Diseases of the Cortex, J. Neurosci. 28, 1709 (2008).

[46] H. R. Wilson and J. D. Cowan, Excitatory and Inhibitory Interactions in Localized Populations of Model Neurons, Biophys. J. 12, 1 (1972).

[47] T. P. Vogels, K. Rajan, and L. F. Abbott, Neural Network Dynamics, Annu. Rev. Neurosci. 28, 357 (2005).

[48] P. Fries, D. Nikolić, and W. Singer, The Gamma Cycle, Trends Neurosci. 30, 309 (2007).

[49] N. Brunel, Dynamics of Sparsely Connected Networks of Excitatory and Inhibitory Spiking Neurons, J. Comput. Neurosci. 8, 183 (2000).

[50] A. Lerchner and P. E. Latham, A Unifying Framework for Understanding State-Dependent Network Dynamics in Cortex, arXiv:1511.00411.

[51] S. di Santo, P. Villegas, R. Burioni, and M. A. Muñoz, Landau-Ginzburg Theory of Cortex Dynamics: Scale-Free Avalanches Emerge at the Edge of Synchronization, Proc. Natl. Acad. Sci. U.S.A. 115, E1356 (2018).

[52] M. Tsodyks, K. Pawleslik, and H. Markram, Neural Networks with Dynamic Synapses, Neural Comput. 10, 821 (1998).

[53] A. Levina, J. M. Herrmann, and T. Geisel, Dynamical Synapses Causing Self-Organized Criticality in Neural Networks, Nat. Phys. 3, 857 (2007).

[54] A. Levina, J. M. Herrmann, and T. Geisel, Phase Transitions Towards Criticality in a Neural System with Adaptive Interactions, Phys. Rev. Lett. 102, 118110 (2009).

[55] T. P. Vogels, H. Sprekeler, F. Zenke, C. Clopath, and W. Gerstner, Inhibitory Plasticity Balances Excitation and Inhibition in Sensory Pathways and Memory Networks, Science 334, 1569 (2011).

[56] F. Effenberger, J. Jost, and A. Levina, Self-Organization in Balanced State Networks by STDP and Homeostatic Plasticity, PLoS Comput. Biol. 11, e1004420 (2015).

[57] S. Bornholdt and T. Rohlf, Topological Evolution of Dynamical Networks: Global Criticality from Local Dynamics, Phys. Rev. Lett. 84, 6114 (2000).

[58] C. Tetzlaff, S. Okujeni, U. Egert, F. Wörgötter, and M. Butz, Self-Organized Criticality in Developing Neuronal Networks, PLoS Comput. Biol. 6, e1001013 (2010).

[59] F. Y. K. Kossio, S. Goedeke, B. van den Akker, B. Ibarz, and R.-M. Memmesheimer, Growing Critical: Self-Organized Criticality in a Developing Neural System, Phys. Rev. Lett. (to be published).

[60] L. de Arcangelis, C. Perrone-Capano, and H. J. Herrmann, Self-Organized Criticality Model for Brain Plasticity, Phys. Rev. Lett. 96, 028107 (2006).
[61] J. A. Bonachela, S. De Franciscis, J. J. Torres, and M. A. Muñoz, Self-Organization without Conservation: Are Neuronal Avalanches Generically Critical?, J. Stat. Mech. 2010, P02015 (2010).

[62] A. A. Costa, M. Copelli, and O. Kinouchi, Can Dynamical Synapses Produce True Self-Organized Criticality?, J. Stat. Mech. 2015, P06004 (2015).

[63] L. M. van Kessenich, L. de Arcangelis, and H. J. Herrmann, Synaptic Plasticity and Neuronal Refractory Time Cause Scaling Behaviour of Neuronal Avalanches, Sci. Rep. 6, 32071 (2016).

[64] J. G. F. Campos, A. de Andrade Costa, M. Copelli, and O. Kinouchi, Correlations Induced by Depressing Synapses in Critically Self-Organized Networks with Quenched Dynamics, Phys. Rev. E 95, 042303 (2017).

[65] V. Hernandez-Urbina and J. M. Herrmann, Self-Organized Criticality via Retro-Synaptic Signals, Front. Phys. 4, 54 (2017).

[66] B. Del Papa, V. Priesemann, and J. Triesch, Criticality Meets Learning: Criticality Signatures in a Self-Organizing Recurrent Neural Network, PLoS One 12, e0178683 (2017).

[67] D. Millman, S. Mihalas, A. Kirkwood, and Ernst Niebur, SelfOrganized Criticality Occurs in Non-conservative Neuronal Networks During “Up” States, Nat. Phys. 6, 801 (2010).

[68] M. Martinello, J. Hidalgo, A. Maritan, S. di Santo, D. Plenz, and M. A. Muñoz, Neutral Theory and Scale-Free Neural Dynamics, Phys. Rev. X 7, 041071 (2017).

[69] A Lazar, G Pipa, and J Triesch, SORN: A Self-Organizing Recurrent Neural Network, Front. Comput. Neurosci. 3, 23 (2009).

[70] A. Litwin-Kumar and B. Doiron, Formation and Maintenance of Neuronal Assemblies through Synaptic Plasticity, Nat. Commun. 5, 5319 (2014).

[71] F. Zenke, E. J. Agnes, and W. Gerstner, Diverse Synaptic Plasticity Mechanisms Orchestrated to Form and Retrieve Memories in Spiking Neural Networks, Nat. Commun. 6, 6922 (2015).

[72] D. Miner and J. Triesch, Plasticity-Driven Self-Organization under Topological Constraints Accounts for Non-random Features of Cortical Synaptic Wiring, PLoS Comput. Biol. 12, e1004759 (2016).

[73] Z. Tosi and J. Beggs, Cortical Circuits from Scratch: A Metaplastic Architecture for the Emergence of Lognormal Firing Rates and Realistic Topology, arXiv:1706.00133.

[74] http://neurodatasharing.bme.gatech.edu/development-data/ html/index.html.

[75] K. Mizuseki, A. Sirota, E. Pastalkova, and G. Buzsáki, Multi-unit Recordings from the Rat Hippocampus Made During Open Field Foraging, CRCNS.org, 2009.

[76] T. J. Blanche and N. V. Swindale, Nyquist Interpolation Improves Neuron Yield in Multiunit Recordings, J. Neurosci. Methods, 155, 81 (2006).

[77] T. E. Harris, The Theory of Branching Processes (Springer, Berlin, 1963).

[78] J. Touboul and A. Destexhe, Power-Law Statistics and Universal Scaling in the Absence of Criticality, Phys. Rev. E 95, 012413 (2017).

[79] A. Levina and V. Priesemann, Subsampling Scaling, Nat. Commun. 8, 15140 (2017). 
[80] J. Wilting and V. Priesemann, Inferring collective dynamical states from widely unobserved systems, Nat. Commun. 9, 2325 (2018).

[81] V. Priesemann, M. Wibral, M. Valderrama, R. Pröpper, M. Le Van Quyen, T. Geisel, J. Triesch, D. Nikolić, and M. H. J. Munk, Spike Avalanches in Vivo Suggest a Driven, Slightly Subcritical Brain State, Front. Syst. Neurosci. 8, 108 (2014).

[82] E. T. Kavalali, The Mechanisms and Functions of Spontaneous Neurotransmitter Release, Nat. Rev. Neurosci. 16, 5 (2015).

[83] M. Lenz, S. Platschek, V. Priesemann, D. Becker, L. M. Willems, U. Ziemann, T. Deller, F. Müller-Dahlhaus, P. Jedlicka, and A. Vlachos, Repetitive Magnetic Stimulation Induces Plasticity of Excitatory Postsynapses on Proximal Dendrites of Cultured Mouse CA1 Pyramidal Neurons, Brain Struct. Funct. 220, 3323 (2015).

[84] C. Haldeman and J. M. Beggs, Critical Branching Captures Activity in Living Neural Networks and Maximizes the Number of Metastable States, Phys. Rev. Lett. 94, 058101 (2005).

[85] C. R. Heathcote, A Branching Process Allowing Immigration, J. R. Stat. Soc. Ser. B 27, 138 (1965).

[86] A. G. Pakes, Branching Processes with Immigration, J. Appl. Probab. 8, 32 (1971).

[87] P. Erdős and A. Rényi, On the Evolution of Random Graphs, Publ. Math. Inst. Hung. Acad. Sci., Ser. A 5, 17 (1960).

[88] L. Hernández-Navarro, J. G. Orlandi, B. Cerruti, E. Vives, and J. Soriano, Dominance of Metric Correlations in TwoDimensional Neuronal Cultures Described through a Random Field Ising Model, Phys. Rev. Lett. 118, 208101 (2017).

[89] Q. Wen, A. Stepanyants, G. N. Elston, A. Y. Grosberg, and D. B. Chklovskii, Maximization of the Connectivity Repertoire as a Statistical Principle Governing the Shapes of Dendritic Arbors, Proc. Natl. Acad. Sci. U.S.A. 106, 12536 (2009).

[90] G. Buzsáki and K. Mizuseki, The Log-Dynamic Brain: How Skewed Distributions Affect Network Operations, Nat. Rev. Neurosci. 15, 264 (2014).

[91] K. B. Hengen, A. Torrado Pacheco, J. N. McGregor, S. D. Van Hooser, and G. G. Turrigiano, Neuronal Firing Rate Homeostasis Is Inhibited by Sleep and Promoted by Wake, Cell 165, 180 (2016).

[92] V. Priesemann and O. Shriki, Can a Time Varying External Drive Give Rise to Apparent Criticality in Neural Systems, PLoS Comput. Biol. 14, e1006081 (2018).

[93] K. D. Harris and T. D. Mrsic-Flogel, Cortical Connectivity and Sensory Coding, Nature (London) 503, 51 (2013).

[94] U. Hasson, J. Chen, and C. J. Honey, Hierarchical Process Memory: Memory as an Integral Component of Information Processing, Trends Cognit. Sci. 19, 304 (2015).

[95] D. J. Felleman and D. C. Van Essen, Distributed Hierarchical Processing in the Primate Cerebral Cortex, Cereb. Cortex 1, 1 (1991).

[96] M. London, A. Roth, L. Beeren, M. Häusser, and P. E. Latham, Sensitivity to Perturbations In Vivo Implies High Noise and Suggests Rate Coding in Cortex, Nature (London) 466, 123 (2010).
[97] J. Wilting and V. Priesemann, On the Ground State of Spiking Network Activity in Mammalian Cortex, arXiv: 1804.07864.

[98] K. Reinhold, A. D Lien, and M. Scanziani, Distinct Recurrent versus Afferent Dynamics in Cortical Visual Processing, Nat. Neurosci. 18, 1789 (2015).

[99] D. Holcman and M. Tsodyks, The Emergence of Up and Down States in Cortical Networks, PLoS Comput. Biol. 2, e23 (2006).

[100] A. A. Costa, L. Brochini, and O. Kinouchi, Self-Organized Supercriticality and Oscillations in Networks of Stochastic Spiking Neurons, Entropy 19, 399 (2017).

[101] P. Bak, C. Tang, and K. Wiesenfeld, Self-Organized Criticality: An Explanation of 1/f Noise, Phys. Rev. Lett. 59, 381 (1987).

[102] S. Zapperi, K. B. Lauritsen, and H. E. Stanley, SelfOrganized Branching Processes: Mean-Field Theory for Avalanches, Phys. Rev. Lett. 75, 4071 (1995).

[103] C. van Vreeswijk and H. Sompolinsky, Chaos in Neuronal Networks with Balanced Excitatory and Inhibitory Activity, Science 274, 1724 (1996).

[104] A. Renart, J. de la Rocha, P. Bartho, L. Hollender, N. Parga, A. Reyes, and K. D. Harris, The Asynchronous State in Cortical Circuits, Science 327, 587 (2010).

[105] D. Harnack, M. Pelko, A. Chaillet, Y. Chitour, and M. C. W. van Rossum, Stability of Neuronal Networks with Homeostatic Regulation, PLoS Comput. Biol. 11, e1004357 (2015).

[106] D. A. Wagenaar, R. Madhavan, J. Pine, and S. M. Potter, Controlling Bursting in Cortical Cultures with ClosedLoop Multi-Electrode Stimulation, J. Neurosci. 25, 680 (2005).

[107] J. Lian, M. Bikson, C. Sciortino, W. C. Stacey, and D. M. Durand, Local Suppression of Epileptiform Activity by Electrical Stimulation in Rat Hippocampus In Vitro, J. Physiol. 547, 427 (2003).

[108] C.-C. Chiang, T. P. Ladas, L. E. Gonzalez-Reyes, and D. M. Durand, Seizure Suppression by High Frequency Optogenetic Stimulation Using In Vitro and In Vivo Animal Models of Epilepsy, Brain Stimul. 7, 890 (2014).

[109] L. Covolan, A.-C. G. de Almeida et al., Effects of Anterior Thalamic Nucleus Deep Brain Stimulation in Chronic Epileptic Rats, PLoS One 9, e97618 (2014).

[110] T. P. Ladas, C.-C. Chiang, L. V. Gonzalez-Reyes, T. Nowak, and D. M. Durand, Seizure Reduction through Interneuron-mediated Entrainment Using Low Frequency Optical Stimulation, Exp. Neurol. 269, 120 (2015).

[111] S. I. Morefield, E. W. Keefer, K. D. Chapman, and G. W. Gross, Drug Evaluations Using Neuronal Networks Cultured on Microelectrode Arrays, Biosens. Bioelectron. 15, 383 (2000).

[112] W. L. Shew, H. Yang, T. Petermann, R. Roy, and D. Plenz, Neuronal Avalanches Imply Maximum Dynamic Range in Cortical Networks at Criticality, J. Neurosci. 29, 15595 (2009).

[113] Y. Penn, M. Segal, and E. Moses, Network Synchronization in Hippocampal Neurons, Proc. Natl. Acad. Sci. U.S.A. 113, 3341 (2016). 
[114] M. Schottdorf, The reconstitution of visual cortical feature selectivity in vitro, $\mathrm{PhD}$ Thesis, Georg-August-Universität (2017).

[115] W. Janke, Statistical Analysis of Simulations: Data Correlations and Error Estimation, in Proceedings of the Winter School "Quantum Simulations of Complex ManyBody Systems: From Theory to Algorithms", edited by
J. Grotendorst, D. Marx, and A. Muramatsu (NIC, Jülich, 2002), Vol. 10, p. 423.

[116] R. M. Corless, G. H. Gonnet, D. E. G. Hare, D. J. Jeffrey, and D. E. Knuth, On the Lambert W Function, Adv. Comput. Math. 5, 329 (1996).

[117] J. Zierenberg, J. Wilting, V. Priesemann, and A. Levina, Finite-Size Effects in Branching Networks (to be published). 